\title{
A single center's experience with pacemaker implantation after the Cox maze procedure for atrial fibrillation
}

\author{
Niv Ad, MD, ${ }^{\mathrm{a}}$ Sari D. Holmes, PhD, ${ }^{\mathrm{b}}$ Rabia Ali, MS, ${ }^{\mathrm{c}}$ Graciela Pritchard, BS, ${ }^{\mathrm{c}}$ and \\ Deborah Lamont, BSN, $\mathrm{RN}^{\mathrm{c}}$
}

\section{ABSTRACT}

Objective: The Cox maze procedure $(\mathrm{CM})$ is safe and effective for all atrial fibrillation (AF) types. A recent randomized trial found alarming rates of pacemaker implantation (PMI) during hospitalization after CM. The purpose of this study was to assess the rate of PMI and its impact on outcomes after CM.

Methods: Incidence of PMI was captured for all CM patients (2005-2015; $\mathrm{N}=739$ ). Data were collected prospectively. Multivariable logistic regression was conducted to determine risk factors for PMI. Propensity score matching was conducted between concomitant $\mathrm{CM}$ patients and patients without surgical ablation since 2011 .

Results: Fifty-two patients (7.0\%) had in-hospital PMI after CM. Most common primary indication for PMI was sick sinus syndrome $(67 \%)$, followed by complete heart block $(23 \%)$ and sinus bradycardia $(10 \%)$. The only risk factor for in-hospital PMI was type of procedure $(P=.020)$. Patients with multiple valve procedures were at greatest risk $(P=.004-.035)$. STS-defined perioperative outcomes were similar for patients with and without in-hospital PMI. Sinus rhythm off antiarrhythmic drugs were similar by PMI. After propensity score matching ( $\mathrm{n}=180$ per group), in-hospital PMI was similar in CM patients and those without surgical ablation $(5 \%$ vs $4 \%, P=.609)$.

Conclusions: This study demonstrated lower incidence of PMI after CM procedures than recently reported. When indicated, PMI was not associated with increased short- or long-term morbidity or inferior freedom from atrial arrhythmia. Efforts to increase surgeon training with the CM procedure and postoperative management awareness are warranted to improve rhythm outcome and minimize adverse events and PMI. (J Thorac Cardiovasc Surg 2017;154:139-46)

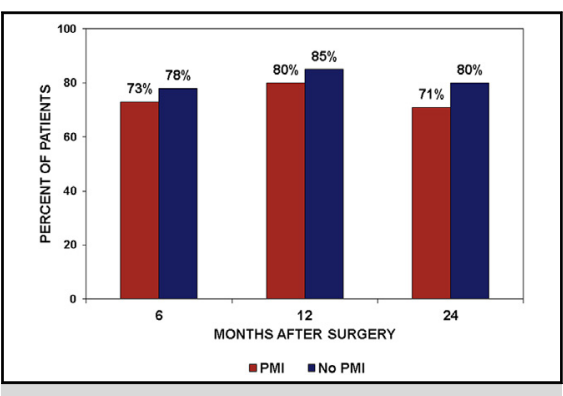

Patients with and without in-hospital PMl in sinus rhythm off $A A D$ during follow-up.

\section{Central Message}

In Cox maze patients, in-hospital PMI was low and not associated with increased morbidity, inferior rhythm outcome, or long-term survival.

\section{Perspective}

Evidence from this study indicated a lower rate of PMI following Cox maze procedures than recently reported. PMI was not associated with increased morbidity or recurrence of atrial arrhythmia. Surgeon training with the Cox maze procedure and postoperative management is warranted, particularly during complicated concomitant procedures, to improve rhythm outcome and minimize adverse events and PMI.

See Editorial Commentary page 147.
The Cox maze (CM) procedure has been performed for more than 25 years and its success when conducted in experienced hands, using either cut and sew technique or ablation devices with proven delivery of transmurality, is well-documented. $^{1-5}$ The CM procedure, performed appropriately, is also demonstrated to be very safe for all

\footnotetext{
From the ${ }^{\mathrm{a}}$ Department of Cardiovascular and Thoracic Surgery, West Virginia University, Morgantown, WVa; ${ }^{\mathrm{b}}$ Heart and Vascular Institute, West Virginia University Medicine, Morgantown, WVa; and ${ }^{\mathrm{c}}$ Department of Cardiac Research, Inova Fairfax Hospital, Falls Church, Va.

Received for publication May 16, 2016; revisions received Nov 15, 2016; accepted for publication Dec 12, 2016; available ahead of print March 2, 2017.

Address for reprints: Niv Ad, MD, Department of Cardiovascular and Thoracic Surgery, West Virginia University, 1 Medical Center Dr, Morgantown, WV 26506 (E-mail: nivadmd14@gmail.com).

$0022-5223 / \$ 36.00$

Copyright (c) 2017 by The American Association for Thoracic Surgery http://dx.doi.org/10.1016/j.jtcvs.2016.12.047
}

atrial fibrillation (AF) types and across operative risk groups. ${ }^{6-10}$ One of the challenges when assessing the efficacy and safety of the procedure is the lack of standardization in the way the procedure is being performed in terms of ablation tools as well as the lesion set. This point is especially important when it comes to lesions applied to the right atrium, with lesion patterns and tissue handling that can lead to relatively high rates of pacemaker implantation (PMI) following the procedure. $^{11}$ There are other potential explanations for

Scanning this QR code will take you to a supplemental figure for this article. 


$$
\begin{aligned}
& \text { Abbreviations and Acronyms } \\
& \text { AAD }=\text { antiarrhythmic drugs } \\
& \text { AF }=\text { atrial fibrillation } \\
& \text { AV }=\text { atrioventricular } \\
& \text { CABG }=\text { coronary artery bypass grafting } \\
& \text { CM }=\text { Cox maze procedure } \\
& \text { CTSN }=\text { Cardiothoracic Surgical Trials Network } \\
& \text { IQR }=\text { interquartile range } \\
& \text { LA }=\text { left atrium } \\
& \text { LOS }=\text { length of stay } \\
& \text { PMI }=\text { pacemaker implantation } \\
& \text { STS }=\text { Society of Thoracic Surgeons }
\end{aligned}
$$

PMI that cannot be explained by technical variability, such as the fact that sinus node dysfunction is recognized to be highly associated with AF and the potential for remodeling of the sinus node tissue and function secondary to AF and antiarrhythmic therapy. ${ }^{12-14}$ The diagnosis of sinus node dysfunction, whether temporary or not, is therefore not unusual after a successful CM procedure simply because a successful procedure would challenge the sinus node to activate the atria that can no longer fibrillate. Sinus node damage secondary to ablative damage during surgical ablation should be extremely rare in a well-performed procedure. However, conduction disturbances, such as atrioventricular block, can be the result of mismanagement of the lateral wall of the right atria or a lesion set that is not consistent with the $\mathrm{CM}$ procedure, together with the indexed concomitant procedure performed. ${ }^{11} \mathrm{~A}$ recent Cardiothoracic Surgical Trials Network (CTSN) randomized trial found alarming rates of PMI during hospitalization after surgical ablation (23 of 133), especially in patients following what was defined by the authors as a $\mathrm{CM}$ procedure. ${ }^{15}$ We were surprised by the unusual rate of PMI in that study and the potential implication on the decision-making associated with surgical ablation of AF. Therefore, we decided to assess our own outcomes, particularly to understand if there are factors, whether preoperative or operative, that may predispose some patients to PMI. In our center, the CM procedure III/IV is performed in a uniform way with regard to lesion set and energy source. ${ }^{2,16,17}$ The purpose of this study was to assess the rate of inhospital PMI for CM III/IV patients in an experienced center, examine factors that may be associated with PMI in those patients, and compare PMI incidence with patients with $\mathrm{AF}$ who did not undergo surgical ablation. We also sought to investigate the potential impact of PMI on early patient outcomes and long-term success rate and survival.

\section{METHODS}

All patients who had a CM III/IV procedure ${ }^{2,16,17}$ from 2005 to 2015 $(\mathrm{N}=739)$ with or without other cardiac procedures were included in this study sample. Patients were excluded if they had previous PMI before surgery $(n=92)$. Data were collected prospectively before and after surgery in our comprehensive AF registry and with the Society of Thoracic Surgeons (STS) institutional database. ${ }^{18}$ Data on rhythm status (24-hour Holter monitoring and electrocardiography), medications, interventions, and clinical events were collected at 3, 6, 9,12,18, and 24 months and then yearly after surgery. Rhythm status was defined according to Heart Rhythm Society guidelines as freedom from atrial arrhythmia $>30$ seconds without use of class I/III antiarrhythmic drugs (AAD). ${ }^{19}$ Data on new PMI after surgery in the same surgical hospitalization was collected on all CM patients. This study was approved by our institutional review board (institutional review board no. 06.022 and 12.055), and patient consent was waived.

\section{Surgical CM Procedure}

All CM III/IV procedures were performed either using cryothermal energy alone (ATS CryoMaze, Medtronic, Minneapolis, Minn; or Frigitronics, CooperSurgical, Inc, Trumbull, Conn; and Cryo1, AtriCure, West Chester, Ohio) or a combination of bipolar radiofrequency (Isolator Synergy Bipolar Ablation System, AtriCure) and cryothermal energy. The lesion pattern in all cases adhered to the CM III/IV lesion set. ${ }^{2,16,17}$

\section{Statistical Analysis}

All data analyses were performed with SPSS Version 17.0 (SPSS Inc., Chicago, Ill) and $\mathrm{R}$ version 3.1.3 (The R Foundation for Statistical Computing, Vienna, Austria). Continuous variables are presented as mean \pm standard deviation or median [interquartile range (IQR)], whereas categorical variables are presented as frequency (percentage). Group differences were evaluated with Student $t$ tests or Mann-Whitney $U$ tests for continuous variables and with $\chi^{2}$ tests or Fisher exact test for categorical variables, as appropriate. Risk factors for length of stay (LOS) were examined with multivariable linear regression with the following covariates: age, EuroSCORE II, ejection fraction, gender, body mass index, duration of AF, left atrium (LA) size, AF type, year of surgery, elective versus nonelective status, and type of procedure (stand-alone CM [reference group], multiple concomitant valves, concomitant single valve, concomitant coronary artery bypass grafting [CABG], concomitant CABG with valve surgery, and other concomitant procedures). Multivariable logistic regression was conducted to determine the risk factors for PMI, including age, AF duration, LA size, type of procedure (multiple concomitant valves [reference group], stand-alone CM, concomitant single valve, concomitant CABG, concomitant CABG with valve surgery, and other concomitant procedures), and year of surgery. Kaplan-Meier analysis with the log-rank test was conducted to compare cumulative survival curves between patients with and without in-hospital PMI.

In the group of patients with a history of atrial arrhythmia since 2011 who had a concomitant CM procedure $(\mathrm{n}=251)$ and patients without surgical ablation $(\mathrm{n}=415)$, propensity score matching was conducted to simulate randomization and improve balance on preoperative characteristics. $^{20,21}$ This time frame was chosen because the STS database began collecting data on in-hospital PMI for all patients in 2011. The control group included all cardiac surgery patients since 2011 with a history of atrial arrhythmia who did not undergo any surgical ablation procedure. Propensity scores were estimated via a logistic model with the R MatchIt package and were matched between groups within a caliper of 0.25 propensity score standard deviations. The variables used to create the propensity score model were selected a priori based on clinical and theoretical relevance and included age, gender, body mass index, diabetes, hypertension, cerebrovascular disease, chronic pulmonary disease, peripheral vascular disease, ejection fraction, preoperative creatinine level, EuroSCORE II, elective status, concomitant valve surgery, and concomitant CABG surgery. Most CM patients were matched to patients in the control group, those without surgical ablation, and balance was improved between the groups (Figure E1). The 180 patients in each group remaining after propensity score matching were used for all outcome analyses comparing 
patients who had CM and those without surgical ablation, particularly to examine in-hospital incidence of PMI.

\section{RESULTS}

The study sample consisted of 739 patients who underwent the full $\mathrm{CM}$ procedure. The mean age was $63.4 \pm 11.1$ years and 238 patients were women $(32 \%)$. The CM procedure was performed concomitant with other surgical procedures in $79 \%$ of patients and as a standalone procedure in $21 \%$ of patients.

Fifty-two patients $(7.0 \%)$ had newly implanted pacemakers during the hospital stay after the CM procedure. The type of in-hospital PMI was dual-chamber in all of these patients and mean time between surgery and PMI was $9.3 \pm 5.5$ days (median $[\mathrm{IQR}]=8$ [6-11] days). The most common primary indication for PMI was sick sinus syndrome $(67 \%)$, followed by complete heart block $(23 \%)$ and sinus bradycardia $(10 \%)$. Patients with inhospital PMI had lower body mass index $(P=.006)$, lower median duration of AF $(P=.047)$, were more likely to be women $(P=.011)$, and were less likely to have hypertension $(P=.017)$ than patients without in-hospital PMI (Table 1). Otherwise, the patients with in-hospital PMI had similar preoperative characteristics as patients without in-hospital PMI. Patients who had 2 or more concomitant valve procedures had the highest total incidence of PMI $(\mathrm{PMI}=14 \%)$. In addition, the group of patients with in-hospital PMI were significantly less likely to have a stand-alone CM procedure compared with patients without in-hospital PMI (10\% vs $22 \%, P=.032)$.

In multivariable analyses (Table 2), the only significant independent association with in-hospital PMI was type of surgical procedure (Wald $=13.44, P=.020$ ), such that patients who had multiple concomitant valve procedures were at greater risk than other surgery categories. Compared with patients with multiple concomitant valve procedures, those with stand-alone CM procedures had 80\% lower odds for in-hospital PMI, those with concomitant single-valve procedures had $57 \%$ lower odds, those with concomitant CABG surgery procedures had $89 \%$ lower odds, and those with concomitant $\mathrm{CABG}$ with valve surgery procedures had $73 \%$ lower odds.

\section{Clinical Outcome}

Patients with in-hospital PMI had similar incidence of STS-defined perioperative complications compared with patients without in-hospital PMI, including deep sternal wound infection ( $0 \%$ vs $0.1 \%, P>.999)$, stroke/transient ischemic attack $(0 \%$ vs $0.9 \%, P>.999)$, prolonged ventilation $>24$ hours $(12 \%$ vs $8 \%, P=.428)$, pneumonia ( $2 \%$ vs $4 \%, P>.999)$, renal failure $(2 \%$ vs $4 \%, P>$ $.999)$, reoperation for bleeding $(0 \%$ vs $3 \%, P=.397)$, readmissions $<30$ days ( $8 \%$ vs $13 \%, P=.386$ ), and operative mortality $<30$ days ( $0 \%$ vs $2 \%, P=.615$ ). However, patients with in-hospital PMI had a longer median hospital LOS than patients without in-hospital PMI (12 [9-15]

TABLE 1. Patient characteristics by in-hospital PMI

\begin{tabular}{|c|c|c|c|}
\hline Characteristic ${ }^{*}$ & No PMI (n = 687) & PMI $(\mathbf{n}=52)$ & $\boldsymbol{P}$ \\
\hline Age (y) & $63.3 \pm 11.1$ & $64.2 \pm 12.0$ & .581 \\
\hline Female & $213(31)$ & $25(48)$ & .011 \\
\hline Body mass index $\left(\mathrm{kg} / \mathrm{m}^{2}\right)$ & $28.4 \pm 5.8$ & $26.1 \pm 5.6$ & .006 \\
\hline Ejection fraction $(\%)$ & $55.4 \pm 10.7$ & $55.8 \pm 10.6$ & .751 \\
\hline Diabetes & $91(13)$ & $8(15)$ & .662 \\
\hline Hypertension & $432(63)$ & $24(46)$ & .017 \\
\hline Peripheral vascular disease & $38(6)$ & $3(6)$ & $>.999$ \\
\hline Previous cerebrovascular accident & $41(6)$ & $6(12)$ & .132 \\
\hline Chronic pulmonary disease & $131(19)$ & $7(14)$ & .317 \\
\hline Duration of $\mathrm{AF}(\mathrm{mo})$ & $24[4-64]$ & $10[3-34]$ & .047 \\
\hline Left atrium size $(\mathrm{cm})$ & $5.1 \pm 1.0$ & $4.9 \pm 0.8$ & .196 \\
\hline Long-standing persistent AF type & $349(51)$ & $23(44)$ & .361 \\
\hline EuroSCORE II ( $\%)$ & $3.6 \pm 4.6$ & $4.2 \pm 4.5$ & .359 \\
\hline Elective status & $598(87)$ & $44(85)$ & .617 \\
\hline Cryothermia only energy source & $418(61)$ & $35(67)$ & .376 \\
\hline \multicolumn{4}{|l|}{ Concomitant procedures } \\
\hline CABG surgery & $61(9)$ & $3(6)$ & .441 \\
\hline Single mitral valve surgery & $171(25)$ & $13(25)$ & .984 \\
\hline Single aortic valve surgery & $47(7)$ & $2(4)$ & .401 \\
\hline Single tricuspid valve surgery & $8(1)$ & $2(4)$ & .107 \\
\hline CABG/valve surgery & $87(13)$ & $5(10)$ & .522 \\
\hline Multiple valve surgery & $107(16)$ & $18(35)$ & $<.001$ \\
\hline Other procedures & $53(8)$ & $4(8)$ & .992 \\
\hline
\end{tabular}

PMI, Pacemaker implantation; $A F$, atrial fibrillation; $C A B G$, coronary artery bypass grafting. *Data presented as mean \pm standard deviation, median [interquartile range], or frequency (percent). 
TABLE 2. Results of multivariable analysis predicting in-hospital PMI

\begin{tabular}{llcc}
\hline \multicolumn{1}{c}{ Predictor } & OR & $\mathbf{9 5 \%}$ CI & $\boldsymbol{P}$ \\
\hline Age (y) & 1.002 & $0.98-1.03$ & .871 \\
Duration of AF (y) & 0.95 & $0.87-1.03$ & .220 \\
Left atrium size (cm) & 0.73 & $0.52-1.03$ & .074 \\
Year of surgery & 0.92 & $0.82-1.03$ & .132 \\
Type of procedure* & & & \\
$\quad$ Stand-alone & 0.20 & $0.07-0.60$ & .004 \\
Single valve & 0.43 & $0.20-0.93$ & .032 \\
CABG & 0.11 & $0.01-0.86$ & .035 \\
CABG/valve surgery & 0.27 & $0.08-0.87$ & .028 \\
Other procedures & 0.35 & $0.10-1.30$ & .118 \\
\hline$P M I$, Par &
\end{tabular}

$P M I$, Pacemaker implantation; $O R$, odds ratio; $C I$, confidence interval; $A F$, atrial fibrillation; $C A B G$, coronary artery bypass grafting. *Reference group $=$ multiple concomitant valve procedures.

days vs $6[4-8]$ days, $P<.001$ ), partly related to the timing of PMI, which was a median of 8 days following the procedure. In addition, longer LOS was significantly associated with higher EuroSCORE II $(t=3.78, P<.001)$, nonelective status $(t=1.96, P=.050)$, and all concomitant procedure types compared with stand-alone procedures $(P=.048$ $P<.001)$ in a multivariable analysis.

During follow-up, patients with an in-hospital PMI did not differ from patients without an in-hospital PMI on return to sinus rhythm off AAD at 6 months $(73 \%$ vs $78 \%$, $P=.515), 12$ months $(80 \%$ vs $85 \%, P=.409)$, and 24 months $(71 \%$ vs $80 \%, P=.265$; Figure 1$)$. There was also no difference between patients receiving in-hospital PMI and those without in-hospital PMI on the proportion of patients who required cardioversions $(23 \%$ vs $20 \%$, $P=.569)$ or ablations ( $8 \%$ vs $6 \%, P=.564)$ during a mean follow-up of $53 \pm 36$ months.

During follow-up, none of the patients with in-hospital PMI had an embolic stroke and only 12 patients without

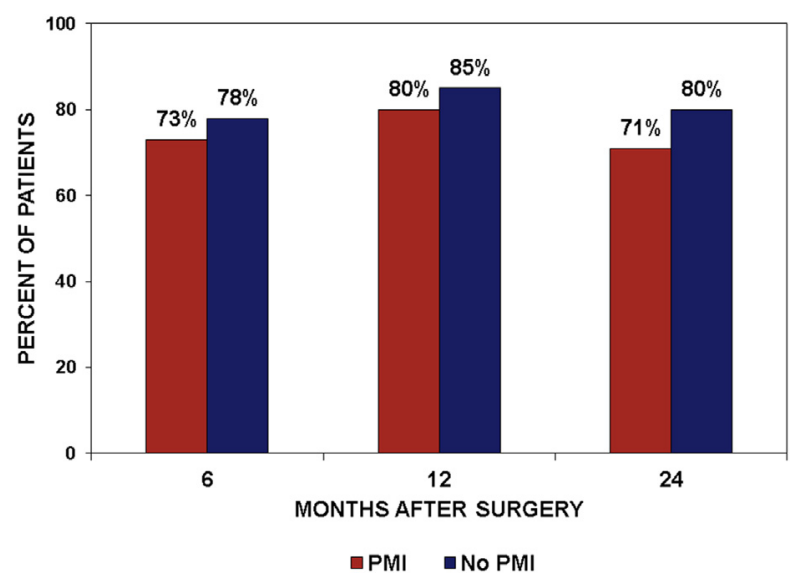

FIGURE 1. Percent of Cox maze patients with and without in-hospital pacemaker implantation (PMI) who returned to sinus rhythm off antiarrhythmic drugs during follow-up.
PMI had embolic strokes $(0 \%$ vs $1.7 \%, P>.999)$. There was no significant difference in percentage of patients who remained on anticoagulation at 6 months $(68 \%$ vs $52 \%, P=.061)$, but more patients with in-hospital PMI were on anticoagulation at 12 months $(50 \%$ vs $34 \%$, $P=.042)$ and 24 months $(50 \%$ vs $32 \%, P=.041)$ after surgery compared with those without in-hospital PMI. However, most patients in both groups (with and without in-hospital PMI) who remained on anticoagulation had clinically appropriate indications (eg, mechanical valve, history of deep vein thrombosis, atrial arrhythmia recurrence) at 6 months $(78 \%$ vs $77 \%, P=.943), 12$ months $(65 \%$ vs $63 \%, P=.871)$, and 24 months $(87 \%$ vs $79 \%$, $P=$.736). Patients with in-hospital PMI also did not differ from patients without in-hospital PMI on survival up to 5 years after surgery $(81.9 \%$ vs $88.9 \%, \log$-rank $=2.8$, $P=.093$; Figure 2).

Pacemaker interrogation reports reviewed during followup indicated that for those with in-hospital PMI, 12 patients had return of intact conduction (ie, no longer pacemakerdependent), 28 patients had not returned to intact conduction, and 12 patients were unknown either because no interrogation reports were available or there was no description of conduction by the electrophysiologist.

\section{Propensity Score-Matched Analyses}

Before propensity score matching, the group of patients with concomitant CM surgery $(\mathrm{n}=251)$ differed from the patients with AF without surgical ablation $(n=415)$ on many preoperative characteristics, including age (65 vs 69 years, $P<.001)$, ejection fraction $(55 \%$ vs $47 \%$, $P<.001)$, EuroSCORE II $(3.9 \%$ vs $8.7 \%, P<.001)$, diabetes $(16 \%$ vs $31 \%, P<.001)$, elective status $(87 \%$ vs $57 \%, P<.001)$, valve procedures $(84 \%$ vs $51 \%$, $P<.001)$, and CABG surgery ( $24 \%$ vs $37 \%, P=.001)$. After propensity score matching to simulate randomization and balance covariates, 180 patients in the $\mathrm{CM}$ group were matched to 180 patients with AF without surgical ablation (Figure E1). There were no significant differences between the groups after matching on any of the preoperative characteristics and the standardized differences were appropriate (Table 3). After matching, the incidence of inhospital PMI was similar in patients with AF who had concomitant $\mathrm{CM}$ and those who did not have surgical ablation $(5 \%$ vs $4 \%, P=.609)$. In a double robust analysis, addition of the $\mathrm{CM}$ procedure also was not associated with greater incidence of in-hospital PMI after controlling for the covariates included in the propensity score model (odds ratio $1.32,95 \%$ confidence interval 0.45-3.85, $P=.612)$.

\section{DISCUSSION}

The results of this study indicate that in patients who undergo a CM III/IV procedure, the incidence of in-hospital 


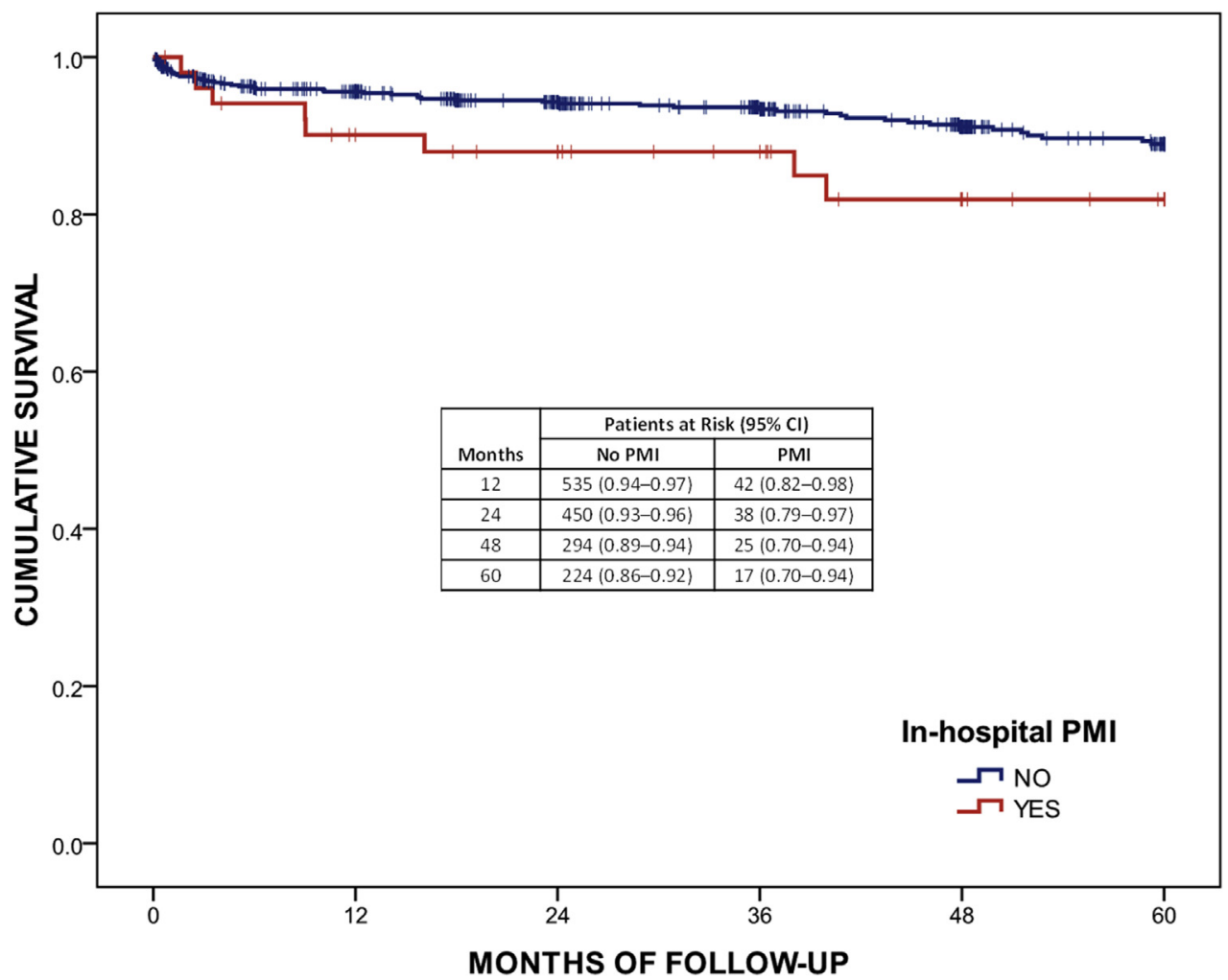

FIGURE 2. Comparison of 5-year survival between patients with and without in-hospital pacemaker implantation (PMI) $(\log -\mathrm{rank}=2.8, P=.093) . C I$, Confidence interval.

PMI was low and was not associated with increased morbidity, inferior rhythm outcome, or differences in long-term survival. Evidence from our study shows that the incidence of in-hospital PMI after a CM procedure is considerably lower than recently reported. ${ }^{15}$ When the $\mathrm{CM}$ procedure is performed, even concomitantly with more complicated multiple valve procedures, a very low incidence of atrioventricular (AV) block was documented, which is probably the result of surgical valve procedures and not with any of the lesions associated with the CM procedure. In addition, propensity score-matched analyses suggest that adding the CM procedure was not associated with excess in-hospital PMI risk. This result was found in patients with comparable preoperative characteristics.

TABLE 3. Patient characteristics for patients with atrial fibrillation with the Cox maze procedure and those without surgical ablation after propensity score matching

\begin{tabular}{|c|c|c|c|c|}
\hline Characteristic & No ablation $(n=180)$ & Cox maze $(n=180)$ & $\boldsymbol{P}$ & St Diffs \\
\hline Age (y) & $68.2 \pm 12.7$ & $67.1 \pm 10.2$ & .365 & -0.096 \\
\hline Female & $61(34)$ & $59(33)$ & .823 & -0.021 \\
\hline Body mass index $\left(\mathrm{kg} / \mathrm{m}^{2}\right)$ & $28.2 \pm 6.0$ & $28.1 \pm 6.1$ & .907 & -0.013 \\
\hline Ejection fraction $(\%)$ & $53.5 \pm 14.2$ & $54.8 \pm 11.0$ & .309 & 0.102 \\
\hline Diabetes & $43(24)$ & $38(21)$ & .528 & -0.071 \\
\hline Hypertension & $129(72)$ & $125(69)$ & .644 & -0.059 \\
\hline Preoperative creatinine level & $1.0[0.8-1.1]$ & $1.0[0.8-1.1]$ & .477 & -0.060 \\
\hline Peripheral vascular disease & $14(8)$ & $14(8)$ & $>.999$ & 0 \\
\hline Cerebrovascular disease & $32(18)$ & $29(16)$ & .673 & -0.053 \\
\hline Chronic pulmonary disease & $55(31)$ & $50(28)$ & .562 & -0.065 \\
\hline EuroSCORE II $(\%)$ & $4.9 \pm 3.8$ & $4.4 \pm 4.5$ & .282 & -0.114 \\
\hline Elective status & $149(83)$ & $149(83)$ & $>.999$ & 0 \\
\hline Concomitant CABG surgery & $60(33)$ & $55(31)$ & .572 & -0.043 \\
\hline Concomitant valve procedures & $135(75)$ & $140(78)$ & .535 & 0.067 \\
\hline
\end{tabular}

Data presented as mean \pm standard deviation, median [interquartile range], or frequency (percent). St Diffs, Standardized differences; CABG, coronary artery bypass grafting. 
In recent years, there has been a growing interest in the rate of PMI following surgical ablation of AF, especially when a biatrial lesion set is performed. ${ }^{11,15}$ Two different reports based on the STS database recognized PMI following surgical ablation and reported slightly higher incidence following surgical ablation combined with mitral valve surgery, but no difference in the rate of postsurgical PMI was identified in stand-alone procedures when comparing surgical ablation performed on and off cardiopulmonary bypass, which probably indicates the same rates of PMI when a biatrial lesion set is performed. ${ }^{22,23}$ The International Society for Minimally Invasive Cardiothoracic Surgery consensus statement clearly showed no increase in the risk for PMI associated with concomitant surgical ablation. ${ }^{24}$

PMI following any cardiac surgical procedures, especially in patients who underwent complex valve surgery, transcatheter aortic valve implantation, or in patients who are elderly, is not uncommon and is a well-accepted outcome among the medical community. ${ }^{25-27}$ However, when it comes to surgical ablation procedures for $\mathrm{AF}$, it is clearly emphasized by some as an especially negative outcome and is readily overstated. The questions to ask in future research are whether the incidence can be managed by implementing training and appropriate techniques and if PMI following surgical ablation is associated with inferior clinical outcome and rhythm control. In the present study, we found that at an experienced center, incidence of in-hospital PMI could be maintained at a low level, which was comparable to patients without surgical ablation for AF. In addition, for patients who underwent the CM III/IV procedure using standardized techniques, in-hospital PMI was not associated with inferior clinical or rhythm outcomes during follow-up.

The incidence of PMI following any surgical ablation is variable and is dependent on patient characteristics with regard to preoperative sinus node dysfunction and surgical technique and manipulation. However, it is also important to standardize the clinical indications for PMI. In our center, patients with postprocedural bradycardia are being paced and atrial electrocardiogram is being captured daily. Therefore, the decision is based on clinical data demonstrating no recovery of sinus node activity, clinically significant conduction blocks or delays, and sinus bradycardia. The impact of PMI should be examined carefully, and in the context of surgical ablation it should always be evaluated beyond the immediate increase in perioperative cost. Although LOS was longer for patients with in-hospital PMI, part of this increase was due to other important factors that would increase LOS anyway, such as gender, EuroSCORE II, elective status, and more complex types of procedures. This issue is important to examine more closely in future research, but ultimately if longer LOS is generally the only perioperative complication associated with in-hospital PMI after surgical ablation, then the impact may not be that critical. In our center, patients who are at risk for PMI are followed daily before the decision and this together with the delayed anticoagulation treatment is well reflected in LOS for these patients. The slight delay in the decision to proceed with PMI may be perceived by some as one of the main reasons for our lower rates of PMI. It is difficult to explain the PMI rate only by extended wait time in patients with bradycardia before the decision for implantation. In the CTSN study, the mean LOS was $13.2 \pm$ 8.8 days for the ablation group and $12.4 \pm 10.3$ days for the control group, similar to the reported LOS for patients with PMI in our study. ${ }^{15}$ We believe that if indeed our postoperative management has such an impact, it would be very important to conduct a controlled study to compare different schemes of postoperative care and decision-making. However, it is important to notice that if a longer wait before PMI had a true impact on the incidence of PMI it would be reflected in the rate of pacemaker dependency during follow-up. In the ABLATE trial, it was reported that approximately $40 \%$ of the patients were still in various degrees of pacemaker dependency after 18 months. ${ }^{28}$ These rates somewhat resemble the data from our study.

In this study, we investigated the incidence of PMI following a full $\mathrm{CM}$ procedure in a relatively large number of patients. We found that the incidence of in-hospital PMI was relatively low, especially when compared with a recent CTSN study. ${ }^{15}$ In our series, the vast majority of patients had PMI due to sinus node dysfunction and severe sinus bradycardia, whereas the CTSN study demonstrated relatively high incidence of AV block or delay as the primary indication. This observation is particularly noteworthy because when it comes to the right atrial lesion set and technique, it is especially important not to place additional lesions and avoid any unnecessary manipulations to the right atrial free wall. The concern when reviewing the CTSN study is that the study protocol allows an additional cavo isthmus line (flutter line) that is not part of the CM procedure and results in significant compartmentalization of the right atrium and conduction delays. ${ }^{11,15}$ It is also especially important to recognize that in our series, despite high rates of sinus rhythm in the early postoperative time frame, the incidence of PMI remained fairly low.

Sinus node dysfunction is associated with AF. ${ }^{12-14,29}$ The role of antiarrhythmic therapy before surgery is also worth investigation, as it may lead to a temporary sinus node dysfunction or severe bradycardia. The $\mathrm{CM}$ procedure should not result in primary sinus node dysfunction if careful application of the lesions is followed. In our study, the major indications for PMI were sick sinus syndrome, heart block, and sinus bradycardia, and more complex valve procedures were associated with greater likelihood of in-hospital PMI. A significant number of our patients were no longer pacemaker-dependent during follow-up $(30 \%)$ and maintained sinus rhythm off AAD $(71 \%$ at 
2 years). This finding is not the first observation of such outcome, although our study was not specifically designed to follow the recovery of the sinus node and the autonomic nervous system. ${ }^{30,31}$ These studies also may suggest that procedures that are associated with higher success in ablating AF may reveal higher rates of sinus node dysfunction, as the heart is no longer allowed to fibrillate and generate heart rate. The concept of success in surgical ablation resulting in sinus node challenge should be investigated further.

\section{CONCLUSIONS}

One of the most important aspects of PMI following the $\mathrm{CM}$ procedure that was addressed in our study is the potential impact on patient outcome during both early and late follow-up. In our study, no such effect was discovered, including morbidity, mortality, rhythm status, and embolic events. PMI following the CM III/IV procedure was definitely associated with increased resource utilization; however, this should be reviewed carefully in the long-term as higher rates of sinus rhythm together with improved quality of life, reduced risk of stroke, and anticoagulation treatment and overall health-related cost. A careful examination into surgeon training and experience with the CM procedure, particularly during complicated concomitant procedures, may well help to improve rhythm outcomes and minimize adverse events, including in-hospital PMI.

\section{Conflict of Interest Statement}

Dr Ad is a consultant for Medtronic, a member of the speaker's bureau for AtriCure, proctor and member of the speaker's bureau for LivaNova, on the advisory board for Nido Surgical, and co-owner for Left Atrial Appendage Occlusion, LLC. All other authors have nothing to disclose with regard to commercial support.

Data were collected and maintained at Inova Fairfax Hospital in Falls Church, Va.

\section{References}

1. Cox JL, Boineau JP, Schuessler RB, Ferguson TB Jr, Cain ME, Lindsay BD, et al. Successful surgical treatment of atrial fibrillation. Review and clinical update. JAMA. 1991;266:1976-80.

2. Damiano RJ Jr, Voeller RK. Surgical and minimally invasive ablation for atrial fibrillation. Curr Treat Options Cardiovasc Med. 2006;8:371-6.

3. Weimar T, Schena S, Bailey MS, Maniar HS, Schuessler RB, Cox JL, et al. The Cox maze procedure for lone atrial fibrillation: a single-center experience over 2 decades. Circ Arrhythm Electrophysiol. 2012;5:8-14.

4. Ad N, Henry L, Friehling T, Wish M, Holmes SD. Minimally invasive standalone Cox-maze procedure for patients with nonparoxysmal atrial fibrillation. Ann Thorac Surg. 2013;96:792-8.

5. Yanagawa B, Holmes SD, Henry L, Hunt S, Ad N. Outcome of concomitant Coxmaze III procedure using an argon-based cryosurgical system: a single-center experience with 250 patients. Ann Thorac Surg. 2013;95:1633-9.

6. Ad N, Holmes SD, Pritchard G, Shuman DJ. Association of operative risk with the outcome of concomitant Cox maze procedure: a comparison of results across risk groups. J Thorac Cardiovasc Surg. 2014;148:3027-33.
7. Ad N, Henry L, Hunt S, Holmes SD, Halpin L. Results of the Cox-maze III/IV procedure in patients over 75 years old who present for cardiac surgery with a history of atrial fibrillation. J Cardiovasc Surg (Torino). 2013;54:281-8.

8. Ad N, Henry LL, Holmes SD, Hunt SL. The impact of surgical ablation for atrial fibrillation in high-risk patients. Ann Thorac Surg. 2012;93:1897-903; discussion 1903-4.

9. Saint LL, Damiano RJ Jr, Cuculich PS, Guthrie TJ, Moon MR, Munfakh NA, et al. Incremental risk of the Cox-maze IV procedure for patients with atria fibrillation undergoing mitral valve surgery. J Thorac Cardiovasc Surg. 2013; 146:1072-7.

10. Henn MC, Lawrance CP, Sinn LA, Miller JR, Schuessler RB, Moon MR, et al. Effectiveness of surgical ablation in patients with atrial fibrillation and aortic valve disease. Ann Thorac Surg. 2015;100:1253-60.

11. Soni LK, Cedola SR, Cogan J, Jiang J, Yang J, Takayama H, et al. Right atrial lesions do not improve the efficacy of a complete left atrial lesion set in the surgical treatment of atrial fibrillation, but they do increase procedural morbidity J Thorac Cardiovasc Surg. 2013;145:356-61; discussion 361-3.

12. Choudhury M, Boyett MR, Morris GM. Biology of the sinus node and its disease. Arrhythm Electrophysiol Rev. 2015;4:28-34.

13. Lee JM, Kalman JM. Sinus node dysfunction and atrial fibrillation: two sides of the same coin? Europace. 2013;15:161-2.

14. Hurle A, Climent V, Sanchez-Quintana D. Sinus node structural changes in patients with long-standing chronic atrial fibrillation. J Thorac Cardiovasc Surg. 2006;131:1394-5.

15. Gillinov AM, Gelijns AC, Parides MK, DeRose JJ Jr, Moskowitz AJ, Voisine P, et al. Surgical ablation of atrial fibrillation during mitral-valve surgery. $N$ Engl J Med. 2015;372:1399-409.

16. Cox JL, Schuessler RB, D’Agostino HJJ, Stone CM, Chang BC, Cain ME, et al The surgical treatment of atrial fibrillation. III. Development of a definitive surgical procedure. J Thorac Cardiovasc Surg. 1991;101:569-83.

17. Cox JL. The surgical treatment of atrial fibrillation. IV. Surgical technique. $J$ Thorac Cardiovasc Surg. 1991;101:584-92.

18. Society of Thoracic Surgeons. The STS surgeon composite technical appendix. February 2015. Available at: http://www.sts.org/sites/default/files/documents/ STS-SurgeonCompositeTechnicalAppendix-UpdatedFeb2015.pdf. Accessed April $29,2016$.

19. Calkins H, Kuck KH, Cappato R, Brugada J, Camm AJ, Chen SA, et al. 2012 HRS/EHRA/ECAS expert consensus statement on catheter and surgical ablation of atrial fibrillation: recommendations for patient selection, procedural techniques, patient management and follow-up, definitions, endpoints, and research trial design. J Interv Card Electrophysiol. 2012;33:171-257.

20. Ho D, Imai K, King G, Stuart E. Matching as nonparametric preprocessing for reducing model dependence in parametric causal inference. Polit Anal. 2007; 15:199-236.

21. Ho D, Imai K, King G, Stuart E. MatchIt: nonparametric preprocessing for parametric causal inference. J Stat Softw. 2011;42:1-28.

22. Ad N, Suri RM, Gammie JS, Sheng S, O’Brien SM, Henry L. Surgical ablation of atrial fibrillation trends and outcomes in North America. J Thorac Cardiovasc Surg. 2012;144:1051-60.

23. Gammie JS, Haddad M, Milford-Beland S, Welke KF, Ferguson TB Jr, O’Brien SM, et al. Atrial fibrillation correction surgery: lessons from the Society of Thoracic Surgeons National Cardiac Database. Ann Thorac Surg. 2008;85:909-14.

24. Ad N, Cheng DC, Martin J, Berglin EE, Chang BC, Doukas G, et al. Surgical ablation for atrial fibrillation in cardiac surgery: a consensus statement of the International Society for Minimally Invasive Cardiothoracic Surgery (ISMICS) 2009. Innovations (Phila). 2010;5:74-83.

25. Kaneko T, Vassileva CM, Englum B, Kim S, Yammine M, Brennan M, et al. Contemporary outcomes of repeat aortic valve replacement: a benchmark for transcatheter valve-in-valve procedures. Ann Thorac Surg. 2015;100:1298-304; discussion 1304

26. David TE, Armstrong S, Maganti M, Ihlberg L. Clinical outcomes of combined aortic root replacement with mitral valve surgery. J Thorac Cardiovasc Surg. 2008; 136:82-7.

27. Siontis GC, Juni P, Pilgrim T, Stortecky S, Bullesfeld L, Meier B, et al Predictors of permanent pacemaker implantation in patients with severe aortic stenosis undergoing TAVR: a meta-analysis. J Am Coll Cardiol. 2014; 64:129-40.

28. Philpott JM, Zemlin CW, Cox JL, Stirling M, Mack M, Hooker RL, et al The ABLATE trial: safety and efficacy of Cox maze-IV using a bipolar radiofrequency ablation system. Ann Thorac Surg. 2015;100:1541-6; discussion 1547-8. 
29. Zhao J, Liu T, Li G. Relationship between two arrhythmias: sinus node dysfunction and atrial fibrillation. Arch Med Res. 2014;45:351-5.

30. Pasic M, Musci M, Siniawski H, Edelmann B, Tedoriya T, Hetzer R. Transient sinus node dysfunction after the Cox-maze III procedure in patients with organic heart disease and chronic fixed atrial fibrillation. J Am Coll Cardiol. 1998;32: 1040-7.

31. Pasic M, Musci M, Siniawski H, Grauhan O, Edelmann B, Tedoriya T, et al. The Cox maze III procedure: parallel normalization of sinus node dysfunction, improvement of atrial function, and recovery of the cardiac autonomic nervous system. J Thorac Cardiovasc Surg. 1999;118:287-95.

Key Words: atrial fibrillation, arrhythmia therapy, arrhythmia, pacemaker

Readers who found these articles interesting may also like to read the following papers found in recent and future issues of our sister publications, Seminars in Thoracic and Cardiovascular Surgery and Operative Techniques in Thoracic and Cardiovascular Surgery!

\section{Acquired: Arrythmia}

Original Submission: Mid-Term Performance of Bipolar Radiofrequency Ablation for Isolated Atrial Fibrillation Through a Right Minithoracotomy. Giuseppe Nasso.

Original Submission: Results of Cryoablation for Atrial Fibrillation Concomitant With Video-Assisted Minimally Invasive Mitral Valve Surgery. Giovanni Marchetto. Semin Thoracic Surg 2016;8(2):271-280 


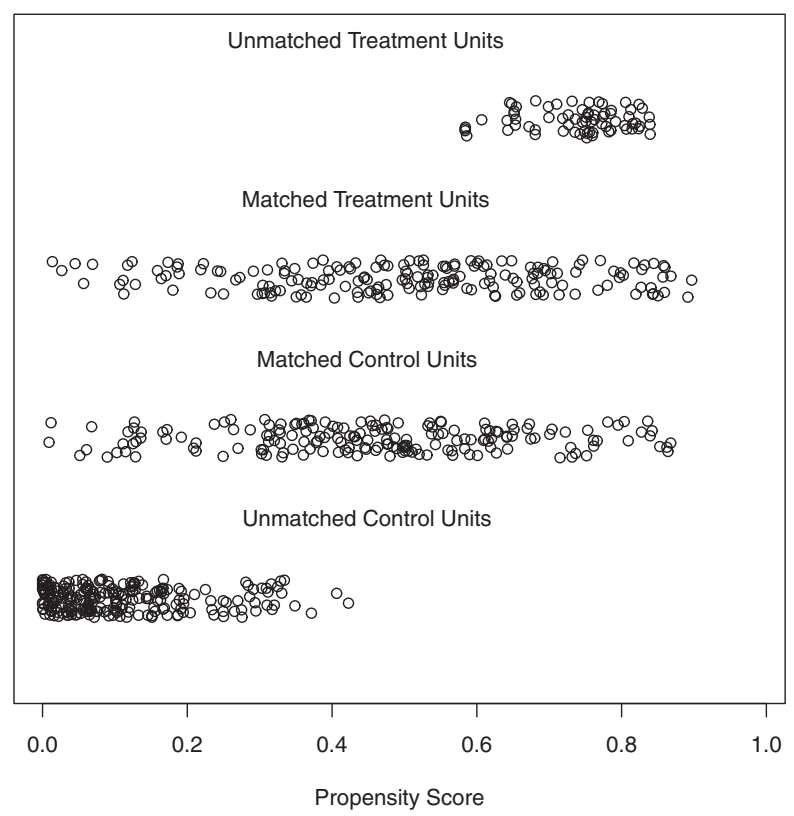

FIGURE E1. Distribution of propensity scores for Cox maze patients (treated units) and patients without surgical ablation (control units) after propensity score matching. 